\title{
Analytic joint spectral radius in a solvable Lie algebra of operators
}

by

\author{
Daniel Beltiţă (Bucureşti)
}

\begin{abstract}
We introduce the concept of analytic spectral radius for a family of operators indexed by some finite measure space. This spectral radius is compared with the algebraic and geometric spectral radii when the operators belong to some finite-dimensional solvable Lie algebra. We describe several situations when the three spectral radii coincide. These results extend well known facts concerning commuting $n$-tuples of operators.
\end{abstract}

1. Introduction. The joint spectral radius ([17]) has recently proved its usefulness in the solution of an important open problem concerning invariant subspaces (cf. [24]; see also [20], [21]). The importance of that concept is also revealed by Theorem 4 of [10], which characterizes the existence of functional calculus with "holomorphic functions" on a polydisk corresponding to a basis in a nilpotent Lie algebra; this extends the natural calculus with polynomials in several non-commuting variables.

Recall that if $\mathcal{X}$ is a complex Banach space and $T \in \mathcal{B}(\mathcal{X})$ is a bounded linear operator on $\mathcal{X}$, then we have the spectral radius formula

$$
r(T):=\sup \{|z| \mid z \in \sigma(T)\}=\lim _{k \rightarrow \infty}\left\|T^{k}\right\|^{1 / k} .
$$

Several multidimensional variants of this equality have been studied. Namely, for arbitrary $T=\left(T_{1}, \ldots, T_{n}\right) \in \mathcal{B}(\mathcal{X})^{n}$ and $p \in[1, \infty]$, in [4], [13] the algebraic spectral radius $\varrho_{p}(T)$ was defined generalizing the right hand side of $(*)$ (see Definition 2.7 below). The middle term of $(*)$ can be directly extended to commuting tuples using the Taylor spectrum or, more generally, to tuples generating a nilpotent Lie algebra, by means of the spectrum introduced in [12]. In this way one gets the geometric spectral radius $r_{p}(T)$ and we have

$$
r_{p}(T) \leq \varrho_{p}(T)
$$

2000 Mathematics Subject Classification: Primary 47A13; Secondary 17B30, 28B05.

Research partially supported by Grant ANSTI no. 6189 GR/25.10.2000. 
whenever $r_{p}(T)$ makes sense (see [4], [16], [13], [7]). Moreover in [7] it is proved that $r_{p}(T)=\varrho_{p}(T)$ if $\operatorname{dim} \mathcal{X}<\infty$. See [14], [4], [23], [13] and [10] for several situations when we have equality in $(* *)$ on infinite-dimensional spaces, thus generalizing $(*)$.

On the other hand, a natural spectrum for finite-dimensional solvable Lie algebras of operators was introduced in [1]. (See [2] for a comparison between this spectrum and the one used in [5], [6] and [15]. Cf. also the monograph [3] for further details.) This allows us to define the geometric spectral radius $r_{p}(T)$ for an $n$-tuple $T \in \mathcal{B}(\mathcal{X})^{n}$ generating a finite-dimensional solvable Lie subalgebra of $\mathcal{B}(\mathcal{X})$ (see Definition 3.10 below). One of the aims of the present paper is to prove that, under these conditions, we have the following improvement of $(* *)$ :

$(* * *) \quad r_{p}(T) \leq \limsup _{w \in \mathbb{C}^{n},\|w\|_{q} \rightarrow \infty} \frac{\log \|\exp (w \cdot T)\|}{\|w\|_{q}} \leq \varrho_{p}(T)$,

where $q \in[1, \infty], 1 / p+1 / q=1$ and $w \cdot T=w_{1} T_{1}+\ldots+w_{n} T_{n}$ for $w=$ $\left(w_{1}, \ldots, w_{n}\right) \in \mathbb{C}^{n}$ (see Corollary 3.11). The middle term of $(* * *)$ will be called the analytic spectral radius of $T$ (see Definition 1.1) and another aim of the present paper is to describe situations when the geometric, analytic and algebraic spectral radii of $T$ coincide (see Theorem 4.1). In particular, we prove generalizations of the following improvement of $(*)$ :

$\forall S \in \mathcal{B}(\mathcal{X}), \quad \sup \{|z| \mid z \in \sigma(S)\}=\limsup _{|w| \rightarrow \infty} \frac{\log \|\exp (w S)\|}{|w|}=\lim _{k \rightarrow \infty}\left\|S^{k}\right\|^{1 / k}$ (see Lemma 3.8).

It is worth noting that (as suggested by [22]) we shall not work actually with finite systems of operators. Instead we study families of operators indexed by some finite measure space $(\mathcal{T}, \mu)$. More precisely, we shall be working with a function

$$
\theta: \mathcal{T} \rightarrow \mathcal{B}(\mathcal{X})
$$

having (at least) the properties that $\sup _{t \in \mathcal{T}}\|\theta(t)\|<\infty$ and for every $x \in \mathcal{X}$ the $\mathcal{X}$-valued function $\theta(\cdot) x$ is Bochner measurable on $(\mathcal{T}, \mu)$. (These assumptions are obvious in the case of $n$-tuples, i.e. when $\mathcal{T}=\{1, \ldots, n\}$ and $\mu$ is the counting measure on $\mathcal{T}$.) Under these conditions it is easily seen that for every $\varphi \in L^{1}(\mathcal{T}, \mu)$ and every $x \in \mathcal{X}$ the function $t \mapsto \varphi(t) \theta(t) x$ is Bochner integrable on $(\mathcal{T}, \mu)$. Moreover there exists $T_{\varphi} \in \mathcal{B}(\mathcal{X})$ such that

$$
\forall x \in \mathcal{X}, \quad T_{\varphi} x=\int_{\mathcal{T}} \varphi(t) \theta(t) x d \mu(t) .
$$

It is convenient to write

$$
T_{\varphi}=: \int_{\mathcal{T}} \varphi \theta d \mu
$$


but we emphasize that this is not intended to mean automatically that $\varphi \theta: \mathcal{T} \rightarrow \mathcal{B}(\mathcal{X})$ is a Bochner integrable $\mathcal{B}(\mathcal{X})$-valued function. We introduce the following:

1.1. Definition. For $p \in[1, \infty]$, the analytic spectral radius of $\theta$, denoted by $\overline{\mathfrak{r}}_{p}(\theta)$, is defined by

$$
\overline{\mathfrak{r}}_{p}(\theta):=\limsup _{\varphi \in L^{q}(\mathcal{T}, \mu),\|\varphi\|_{q} \rightarrow \infty} \frac{\log \left\|\exp \left(\int_{\mathcal{T}} \varphi \theta d \mu\right)\right\|}{\|\varphi\|_{q}},
$$

where $q \in[1, \infty], 1 / p+1 / q=1$. Similarly, the local analytic spectral radius of $\theta$ at $x \in \mathcal{X}$ is defined by

$$
\overline{\mathfrak{r}}_{p}(\theta, x):=\limsup _{\varphi \in L^{q}(\mathcal{T}, \mu),\|\varphi\|_{q} \rightarrow \infty} \frac{\log \left\|\left(\exp \left(\int_{\mathcal{T}} \varphi \theta d \mu\right)\right) x\right\|}{\|\varphi\|_{q}} .
$$

Obviously the analytic spectral radius reduces to the middle term of $(* * *)$ when $\mathcal{T}=\{1, \ldots, n\}, \mu$ is the counting measure on $\mathcal{T}$ and $\theta(j)=T_{j}$ for $j=1, \ldots, n$.

Now we can outline the structure of the present paper. In Section 2 we compare the analytic spectral radius with the algebraic one. In Section 3 we define the Cartan-Taylor spectrum and the geometric spectral radius for a function $\theta$ as above which takes values in some finite-dimensional solvable Lie algebra of operators. Then we compare the three spectral radii: the algebraic, analytic and geometric one (Theorem 3.9). Finally, in Section 4 we describe some situations when these spectral radii coincide (Theorem 4.1). Except for some notations introduced in [1], we use the familiar notation from operator theory.

Finally, I should like to express many thanks to A. A. Dosiev and Yu. V. Turovskil for sending me the preprints of [10], [20] and [21]. Theirs is also the idea to call the spectrum introduced in [1] "Cartan-Taylor" (cf. $[25])$.

2. Analytic vs. algebraic spectral radius. Throughout this section we denote by $(\mathcal{T}, \mu)$ a finite measure space. For every positive integer $k$ and every $\varphi \in L^{1}(\mathcal{T}, \mu)$ we define

$$
\varphi_{[k]}:=\underbrace{\varphi \otimes \ldots \otimes \varphi}_{k \text { times }} \in L^{1}(\mathcal{T} \times \ldots \times \mathcal{T}, \mu \otimes \ldots \otimes \mu),
$$

so $\varphi_{[k]}\left(t_{1}, \ldots, t_{k}\right)=\varphi\left(t_{1}\right) \ldots \varphi\left(t_{k}\right)$ almost everywhere on $\mathcal{T} \times \ldots \times \mathcal{T}$ with respect to $\mu \otimes \ldots \otimes \mu$. Let $\mathcal{X}$ be a complex Banach space. In this section we assume that $\theta: \mathcal{T} \rightarrow \mathcal{B}(\mathcal{X})$ is a function with the following properties:

(i) $\theta$ is bounded, i.e. $\sup \{\|\theta(t)\| \mid t \in \mathcal{T}\}<\infty$.

(ii) For every $x \in \mathcal{X}$, the function $\theta(\cdot) x: \mathcal{T} \rightarrow \mathcal{X}$ is Bochner measurable. 
Then it easily follows from [19] that for every positive integer $k$ and every $x \in \mathcal{X}$, the function

$$
\theta_{[k]}(\cdot) x: \mathcal{T} \times \ldots \times \mathcal{T} \rightarrow \mathcal{X}
$$

is Bochner integrable with respect to $\mu \otimes \ldots \otimes \mu$, where

$$
\theta_{[k]}: \mathcal{T} \times \ldots \times \mathcal{T} \rightarrow \mathcal{B}(\mathcal{X}), \quad\left(t_{1}, \ldots, t_{k}\right) \mapsto \theta\left(t_{1}\right) \ldots \theta\left(t_{k}\right) .
$$

2.1. Remark. For $\theta$ to have the properties (i) and (ii) it suffices that one of the following conditions is satisfied:

$1^{\circ} \mathcal{T}$ is a finite set and $\mu$ is the counting measure on $\mathcal{T}$.

$2^{\circ}$ There exists a finite-dimensional subspace $\mathcal{U}$ of $\mathcal{B}(\mathcal{X})$ such that $\theta(t) \in \mathcal{U}$ for every $t \in \mathcal{T}$, and $\theta$ is a bounded measurable ( $\mathcal{U}$-valued) function on $(\mathcal{T}, \mu)$.

$3^{\circ} \mathcal{T}$ is a compact separable topological space, $\mu$ is a Radon measure on $\mathcal{T}$ and $\theta: \mathcal{T} \rightarrow \mathcal{B}(\mathcal{X})$ is continuous with respect to the norm operator topology on $\mathcal{B}(\mathcal{X})$.

Now let us return to the general situation when $\theta$ has the properties (i) and (ii). Then it is easily seen that for every $k \geq 1, x \in \mathcal{X}$ and $\varphi \in L^{1}(\mathcal{T}, \mu)$, the $\mathcal{X}$-valued function $\varphi_{[k]}(\cdot) \theta_{[k]}(\cdot) x$ is Bochner integrable on $\mathcal{T} \times \ldots \times \mathcal{T}$ with respect to $\mu \otimes \ldots \otimes \mu$. Moreover, by the Fubini theorem, for every $k \geq 1$ we have

$$
\left(T_{\varphi}\right)^{k} x=\int_{\mathcal{T} \times \ldots \times \mathcal{T}} \varphi_{[k]}\left(t_{1}, \ldots, t_{k}\right) \theta_{[k]}\left(t_{1}, \ldots, t_{k}\right) x d(\mu \otimes \ldots \otimes \mu)\left(t_{1}, \ldots, t_{k}\right)
$$

for every $x \in \mathcal{X}$, where $T_{\varphi} \in \mathcal{B}(\mathcal{X})$ is the one from (1). This allows us to introduce the following notation generalizing (2):

$$
\forall k \geq 1, \quad\left(T_{\varphi}\right)^{k}=: \quad \int_{\mathcal{T} \times \ldots \times \mathcal{T}} \varphi_{[k]} \theta_{[k]} d(\mu \otimes \ldots \otimes \mu) .
$$

Now the following fact follows immediately from (2) and (3).

2.2. Lemma. For every $\varphi \in L^{1}(\mathcal{T}, \mu)$ we have

$$
\exp \left(\int_{\mathcal{T}} \varphi \theta d \mu\right)=\sum_{k=0}^{\infty} \frac{1}{k !} \int_{\mathcal{T} \times \ldots \times \mathcal{T}} \varphi_{[k]} \theta_{[k]} d(\mu \otimes \ldots \otimes \mu),
$$

where the series is norm convergent in $\mathcal{B}(\mathcal{X})$. (The first term of the series equals 1 by definition.)

Now let us remark that the properties (i) and (ii) together with the finiteness of the measure $\mu$ imply that for every $k \geq 1, x \in \mathcal{X}$ and $p \in[1, \infty]$ we have

$$
\left\|\theta_{[k]}(\cdot) x\right\| \in L^{p}(\mathcal{T} \times \ldots \times \mathcal{T}, \mu \otimes \ldots \otimes \mu) .
$$

We denote by $\left\|\theta_{[k]}(\cdot) x\right\|_{p}$ the $L^{p}$-norm of $\left\|\theta_{[k]}(\cdot) x\right\|$. Then we can introduce the following concept. 
2.3. Definition. For $p \in[1, \infty]$ we define the algebraic spectral radius of $\theta$ by

$$
\varrho_{p}(\theta):=\limsup _{k \rightarrow \infty}\left(\sup _{\|x\|=1}\left\|\theta_{[k]}(\cdot) x\right\|_{p}\right)^{1 / k},
$$

and the local algebraic spectral radius of $\theta$ at $x \in \mathcal{X}$ by

$$
\varrho_{p}(\theta, x):=\limsup _{k \rightarrow \infty}\left\|\theta_{[k]}(\cdot) x\right\|_{p}^{1 / k} .
$$

2.4. REMARK. (a) If $\mathcal{T}=\{1, \ldots, n\}$ and $\mu$ is the counting measure on $\mathcal{T}$ then $\varrho_{p}(\theta)$ coincides with the algebraic spectral radius $\varrho_{p}(T)$ of the $n$-tuple $T=(\theta(1), \ldots, \theta(n))$ as defined in [4] (see also [7] and Definition 2.7 below).

(b) If $p=\infty, \mathcal{T}$ is an arbitrary set, $\mu$ is the counting (i.e. cardinal) measure on $\mathcal{T}$ and $\theta: \mathcal{T} \rightarrow \mathcal{B}(\mathcal{X})$ has only the property (i) then $\varrho_{\infty}(\theta)$ and $\varrho_{\infty}(\theta, x)$ can also be defined by the above formulas. In this situation the algebraic spectral radius was used in [24], [20], [21], [10] etc. In the same situation, the local algebraic spectral radius was used in [11] and [21].

For the sake of completeness we state the following auxiliary fact.

2.5. Lemma. Let $p, q \in[1, \infty], 1 / p+1 / q=1$. If $(\mathcal{S}, \nu)$ is a measure space, $\varphi \in L^{p}(\mathcal{S}, \nu)$ and $y: \mathcal{S} \rightarrow \mathcal{X}$ is a Bochner integrable function such that $\|y(\cdot)\| \in L^{q}(\mathcal{S}, \nu)$ then $\varphi y$ is a Bochner integrable $\mathcal{X}$-valued function and $\|\varphi y\|_{1} \leq\|\varphi\|_{p} \cdot\|y\|_{q}$.

The main result of this section is the following.

2.6. Proposition. Let $(\mathcal{T}, \mu)$ be a finite measure space and $\theta: \mathcal{T} \rightarrow$ $\mathcal{B}(\mathcal{X})$ be a function with the properties (i) and (ii) above. Then for every $p \in[1, \infty]$ we have

$$
\overline{\mathfrak{r}}_{p}(\theta) \leq \varrho_{p}(\theta)
$$

and

$$
\overline{\mathfrak{r}}_{p}(\theta, x) \leq \varrho_{p}(\theta, x) \quad \text { for every } x \in \mathcal{X} .
$$

Proof. First note that for the second inequality it suffices to consider the case $\|x\|=1$. We prove both inequalities simultaneously and denote by $\mathcal{R}$ (respectively $\mathcal{L}$ ) the right (respectively left) hand side of one of them. So we have to prove $\mathcal{L} \leq \mathcal{R}$.

For every $k \geq 1$ set $M_{k}:=\sup _{\|x\|=1}\left\|\theta_{[k]}(\cdot) x\right\|_{p}$ for the first inequality, and $M_{k}:=\left\|\theta_{[k]}(\cdot) x\right\|_{p}$ for the second. Then

$$
\mathcal{R}=\limsup _{k \rightarrow \infty} M_{k}^{1 / k} .
$$

Hence for each $\varepsilon>0$ there exists a constant $C \geq 1$ such that

$$
\forall k \geq 1, \quad M_{k} \leq C(\mathcal{R}+\varepsilon)^{k} .
$$


Now let $\varphi \in L^{q}(\mathcal{T}, \mu), \varphi \not \equiv 0$, and $x \in \mathcal{X},\|x\|=1$ (where $q \in[1, \infty]$, $1 / p+1 / q=1)$. By Lemma 2.2 we obviously have

$$
\begin{aligned}
\left\|\left(\exp \left(\int_{\mathcal{T}} \varphi \theta d \mu\right)\right) x\right\| & =\left\|\sum_{k=0}^{\infty} \frac{1}{k !} \int_{\mathcal{T} \times \ldots \times \mathcal{T}} \varphi_{[k]}(\cdot) \theta_{[k]}(\cdot) x d(\mu \otimes \ldots \otimes \mu)\right\| \\
& \leq \sum_{k=0}^{\infty} \frac{1}{k !}\left\|\varphi_{[k]}(\cdot) \theta_{[k]}(\cdot) x\right\|_{1} .
\end{aligned}
$$

By Lemma 2.5 and the definition of $M_{k}$, we get

$$
\left\|\left(\exp \left(\int_{\mathcal{T}} \varphi \theta d \mu\right)\right) x\right\| \leq \sum_{k=0}^{\infty} \frac{1}{k !}\left\|\varphi_{[k]}(\cdot)\right\|_{q}\left\|\theta_{[k]}(\cdot) x\right\|_{p} \leq \sum_{k=0}^{\infty} \frac{1}{k !}\left\|\varphi_{[k]}(\cdot)\right\|_{q} \cdot M_{k}
$$

But obviously $\left\|\varphi_{[k]}(\cdot)\right\|_{q}=\|\varphi\|_{q}^{k}$ for each $k$. Hence by (4) one gets

$$
\left\|\left(\exp \left(\int_{\mathcal{T}} \varphi \theta d \mu\right)\right) x\right\| \leq \sum_{k=0}^{\infty} \frac{1}{k !}\|\varphi\|_{q}^{k} \cdot C(\mathcal{R}+\varepsilon)^{k}=C \exp \left(\|\varphi\|_{q}(\mathcal{R}+\varepsilon)\right),
$$

so

$$
\frac{\log \left\|\left(\exp \left(\int_{\mathcal{T}} \varphi \theta d \mu\right)\right) x\right\|}{\|\varphi\|_{q}} \leq \frac{\log C}{\|\varphi\|_{q}}+\mathcal{R}+\varepsilon
$$

In view of the definition of $\mathcal{L}$, the above inequality implies $\mathcal{L} \leq \mathcal{R}+\varepsilon$. Since $\varepsilon>0$ was arbitrary, we get $\mathcal{L} \leq \mathcal{R}$, as desired.

In order to state a corollary of Proposition 2.6 we need the following special case of Definition 2.3 (see Remark 2.4(a)). For a finite family $\left(a_{i}\right)_{i \in I}$ of complex numbers we define as usual

$$
\left\|\left(a_{i}\right)_{i \in I}\right\|_{p}= \begin{cases}\max _{i \in I}\left|a_{i}\right| & \text { if } p=\infty, \\ \left(\sum_{i \in I}\left|a_{i}\right|^{p}\right)^{1 / p} & \text { if } 1 \leq p<\infty .\end{cases}
$$

Moreover for any positive integers $k$ and $n$ we denote by $F(k, n)$ the set of all functions $\iota:\{1, \ldots, k\} \rightarrow\{1, \ldots, n\}$.

2.7. Definition. Let $T=\left(T_{1}, \ldots, T_{n}\right) \in \mathcal{B}(\mathcal{X})^{n}$. Then for $p \in[1, \infty]$ we define the algebraic spectral radius ([4], [13]) of $T$ by

$$
\varrho_{p}(T):=\limsup _{k \rightarrow \infty}\left(\sup _{\|x\|=1}\left\|\left(\left\|T_{\iota(1)} \ldots T_{\iota(k)} x\right\|\right)_{\iota \in F(k, n)}\right\|_{p}\right)^{1 / k},
$$

and the local algebraic spectral radius of $T$ at $x \in \mathcal{X}$ by

$$
\varrho_{p}(T, x):=\limsup _{k \rightarrow \infty}\left(\left\|\left(\left\|T_{\iota(1)} \ldots T_{\iota(k)} x\right\|\right)_{\iota \in F(k, n)}\right\|_{p}\right)^{1 / k} .
$$

Now we can state: 
2.8. Corollary. Let $T \in \mathcal{B}(\mathcal{X})^{n}$ and $p, q \in[1, \infty], 1 / p+1 / q=1$. Then

$$
\overline{\mathfrak{r}}_{p}(T)=\limsup _{w \in \mathbb{C}^{n},\|w\|_{q} \rightarrow \infty} \frac{\log \|\exp (w \cdot T)\|}{\|w\|_{q}} \leq \varrho_{p}(T)
$$

and

$$
\overline{\mathfrak{r}}_{p}(T, x)=\limsup _{w \in \mathbb{C}^{n},\|w\|_{q} \rightarrow \infty} \frac{\log \|(\exp (w \cdot T)) x\|}{\|w\|_{q}} \leq \varrho_{p}(T, x)
$$

for every $x \in \mathcal{X}$.

Proof. Apply Proposition 2.6 for $\mathcal{T}=\{1, \ldots, n\}, \mu$ the counting measure on $\mathcal{T}$ and $\theta: \mathcal{T} \rightarrow \mathcal{B}(\mathcal{X}), \theta(j)=T_{j}$ for $j=1, \ldots, n$.

3. Geometric vs. analytic spectral radius. In this section we are working in a framework which is a special case of the one from Section 2. Namely we use the following objects throughout the present section:

(j) $\mathcal{G}$ is a complex finite-dimensional solvable Lie subalgebra of $\mathcal{B}(\mathcal{X})$;

(jj) $(\mathcal{T}, \mu)$ is a finite measure space;

(jjj) $\theta$ is a bounded measurable $\mathcal{G}$-valued function on $(\mathcal{T}, \mu)$.

We assume moreover that the Banach space $\mathcal{X}$ is non-zero. By Remark $2.1\left(2^{\circ}\right)$, the function $\theta$ also has the properties (i) and (ii) from the beginning of Section 2. In particular, Proposition 2.6 holds in the present framework described by $(\mathrm{j})-(\mathrm{jjj})$.

First of all we define the spectrum and the geometric spectral radius of $\theta$. To this end we use the notations of [1]. The following definition is suggested by Corollary 2.7 of $[1]$.

3.1. Definition. The Cartan-Taylor spectrum of $\theta$ is defined by

$$
\sigma(\theta):=\{\lambda \circ \theta \mid \lambda \in \Sigma(\mathcal{G})\} .
$$

3.2. REMARK. The definition of $\sigma(\theta)$ does not depend on $\mathcal{G}$ in the sense that, if $\mathcal{G}_{1}$ is another finite-dimensional solvable Lie subalgebra of $\mathcal{B}(\mathcal{X})$ such that $\theta(t) \in \mathcal{G}_{1}$ for each $t \in \mathcal{T}$, then

$$
\left\{\lambda \circ \theta \mid \lambda \in \Sigma\left(\mathcal{G}_{1}\right)\right\}=\{\lambda \circ \theta \mid \lambda \in \Sigma(\mathcal{G})\} .
$$

Indeed, we have $\{\theta(t) \mid t \in \mathcal{T}\} \subseteq \mathcal{G}_{1} \cap \mathcal{G}$ and $\Sigma\left(\mathcal{G}_{1} \cap \mathcal{G}\right)=\left.\Sigma\left(\mathcal{G}_{1}\right)\right|_{\mathcal{G}_{1} \cap \mathcal{G}}=$ $\left.\Sigma(\mathcal{G})\right|_{\mathcal{G}_{1} \cap \mathcal{G}}$ by Theorem 2.6 of [1]. So both sides of (5) equal $\{\lambda \circ \theta \mid \lambda \in$ $\left.\Sigma\left(\mathcal{G}_{1} \cap \mathcal{G}\right)\right\}$.

3.3. Remark. For each $p \in[1, \infty], \sigma(\theta)$ is a compact non-empty subset of $L^{p}(\mathcal{T}, \mu)$. Indeed, $\sigma(\theta) \subseteq L^{p}(\mathcal{T}, \mu)$ by the property (jjj) of $\theta$ since $\|\lambda\| \leq 1$ for $\lambda \in \Sigma(\mathcal{G})$ (see Corollary 4 of $\S 27$ in the monograph [3]) and the measure $\mu$ is finite. Moreover $\Sigma(\mathcal{G})$ is a compact non-empty subset of $\widehat{\mathcal{G}}$ by Corollary 2.8 of [1] and $\sigma(\theta)$ is the image of $\Sigma(\mathcal{G})$ under the linear map $\lambda \mapsto \lambda \circ \theta$ defined on the finite-dimensional vector space $\widehat{\mathcal{G}}$. 
The above remark allows us to introduce the following concept.

3.4. Definition. For $p \in[1, \infty]$ we define the geometric spectral radius of $\theta$ by

$$
r_{p}(\theta):=\sup \left\{\|\zeta\|_{p} \mid \zeta \in \sigma(\theta)\right\}
$$

(where $\|\cdot\|_{p}$ denotes the norm of $L^{p}(\mathcal{T}, \mu)$ ).

Now we can prove an auxiliary fact which can be viewed as a kind of spectral mapping theorem.

3.5. Lemma. (a) For $\theta$ as above and $\psi \in L^{1}(\mathcal{T}, \mu)$ we have

$$
\sigma\left(\int_{\mathcal{T}} \psi \theta d \mu\right)=\left\{\int_{\mathcal{T}} \psi \zeta d \mu \mid \zeta \in \sigma(\theta)\right\} .
$$

(b) Let $\mathcal{E}$ be a finite-dimensional nilpotent Lie algebra and $\mathcal{V}$ a finitedimensional subspace of the enveloping algebra $U(\mathcal{E})$. Let $\omega$ be a $\mathcal{V}$-valued measurable function on $(\mathcal{T}, \mu)$ which is bounded (with respect to any norm on $\mathcal{V}$ ). Then for every representation $\varrho: \mathcal{E} \rightarrow \mathcal{B}(\mathcal{X})$ (naturally extended to a morphism $U(\mathcal{E}) \rightarrow \mathcal{B}(\mathcal{X})$ denoted also by $\varrho)$ we have

$$
\sigma\left(\int_{\mathcal{T}} \varrho \circ \omega d \mu\right)=\left\{\int_{\mathcal{T}} \lambda \circ \omega d \mu \mid \lambda \in \sigma(\varrho)\right\} .
$$

Proof. (a) Recall that $\int_{\mathcal{T}} \psi \theta d \mu$ is an element of $\mathcal{G}$, so we can compute its spectrum by Corollary 2.7 of [1]:

$$
\sigma\left(\int_{\mathcal{T}} \psi \theta d \mu\right)=\left\{\lambda\left(\int_{\mathcal{T}} \psi \theta d \mu\right) \mid \lambda \in \Sigma(\mathcal{G})\right\}=\left\{\int_{\mathcal{T}} \psi \cdot(\lambda \circ \theta) d \mu \mid \lambda \in \Sigma(\mathcal{G})\right\} .
$$

Now the desired equality follows from Definition 3.1.

(b) As above, note that $e:=\int_{\mathcal{T}} \omega d \mu$ is an element of $\mathcal{V}$ and

$$
T:=\int_{\mathcal{T}} \varrho \circ \omega d \mu=\varrho\left(\int_{\mathcal{T}} \omega d \mu\right)=\varrho(e) .
$$

Now the spectrum of $T=\varrho(e)$ can be computed by the results of [12] (see either Corollary 0.2 of [1] or Corollary 2.6.7 in [15]):

$$
\begin{aligned}
\sigma(T) & =\{\lambda(e) \mid \lambda \in \sigma(\varrho)\}=\left\{\lambda\left(\int_{\mathcal{T}} \omega d \mu\right) \mid \lambda \in \sigma(\varrho)\right\} \\
& =\left\{\int_{\mathcal{T}} \lambda \circ \omega d \mu \mid \lambda \in \sigma(\varrho)\right\} .
\end{aligned}
$$

The following auxiliary result is suggested by Lemma 2 of [9].

3.6. Lemma. Let $p, q \in[1, \infty], 1 / p+1 / q=1$. For every $\varepsilon>0$ there exists $\psi \in L^{q}(\mathcal{T}, \mu)$ such that $\|\psi\|_{q}=1$ and

$$
r_{p}(\theta)-\varepsilon \leq r\left(\int_{\mathcal{T}} \psi \theta d \mu\right) \leq r_{p}(\theta)
$$


Proof. Since $\sigma(\theta)$ is a compact non-empty subset of $L^{p}(\mathcal{T}, \mu)$ (see Remark 3.3), there exists $\gamma \in \sigma(\theta)$ such that $\|\gamma\|_{p}=r_{p}(\theta)$. Then choose $\psi \in L^{q}(\mathcal{T}, \mu)$ such that

$$
\|\psi\|_{q}=1 \quad \text { and } \quad\|\gamma\|_{p}-\varepsilon \leq\left|\int_{\mathcal{T}} \psi \gamma d \mu\right| \leq\|\gamma\|_{p} .
$$

By Lemma 3.5(a) we have

$$
\sigma\left(\int_{\mathcal{T}} \psi \theta d \mu\right)=\left\{\int_{\mathcal{T}} \psi \zeta d \mu \mid \zeta \in \sigma(\theta)\right\} .
$$

In particular $\int_{\mathcal{T}} \psi \gamma d \mu \in \sigma\left(\int_{\mathcal{T}} \psi \theta d \mu\right)$, so by (6) we get $r\left(\int_{\mathcal{T}} \psi \theta d \mu\right) \geq\|\gamma\|_{p}-\varepsilon$ $=r_{p}(\theta)-\varepsilon$.

On the other hand, by (7) and Hölder's inequality we have

$$
\begin{aligned}
r\left(\int_{\mathcal{T}} \psi \theta d \mu\right) & =\sup \left\{\left|\int_{\mathcal{T}} \psi \zeta d \mu\right| \mid \zeta \in \sigma(\theta)\right\} \\
& \leq \sup \left\{\|\psi\|_{q}\|\zeta\|_{p} \mid \zeta \in \sigma(\theta)\right\}=r_{p}(\theta)
\end{aligned}
$$

where the last equality follows from Definition 3.4 because $\|\psi\|_{q}=1$.

3.7. REMARK. The conclusion of the above lemma also holds for $\varepsilon=0$ if we have the natural isometric isomorphism $\left(L^{p}(\mathcal{T}, \mu)\right)^{*} \cong L^{q}(\mathcal{T}, \mu)$. (This happens e.g. if $p<\infty$ or if $\mathcal{T}$ is a finite set and $\mu$ is the counting measure on $\mathcal{T}$.)

For the sake of completeness we also prove the following fact (see also Corollary 9 of $\S 3$ in the monograph [8]).

3.8. Lemma. For every $S \in \mathcal{B}(\mathcal{X})$ we have

$$
r(S)=\limsup _{|z| \rightarrow \infty} \frac{\log \|\exp (z S)\|}{|z|}=\lim _{k \rightarrow \infty}\left\|S^{k}\right\|^{1 / k} .
$$

Proof. In view of the well known spectral radius formula (see $(*)$ in the Introduction), it suffices to prove the following inequalities:

$$
r(S) \leq \limsup _{|z| \rightarrow \infty} \frac{\log \|\exp (z S)\|}{|z|} \leq \lim _{k \rightarrow \infty}\left\|S^{k}\right\|^{1 / k}
$$

The second inequality follows from Corollary 2.8 for $n=1$. The first inequality can be deduced from the following well known fact:

(9) $\forall R \in \mathcal{B}(\mathcal{X}), \quad \sup \{\operatorname{Re} w \mid w \in \sigma(R)\}=\limsup _{0<t \rightarrow \infty} \frac{\log \|\exp (t R)\|}{t}$.

Indeed, choose $\beta \in \sigma(S)$ with $|\beta|=r(S)$. Moreover let $u \in \mathbb{C}$ be such that $|\beta|=u \beta$ and $|u|=1$. Then $|\beta| \in \sigma(u S)$ and $r(u S)=r(S)=|\beta|$, because 


$$
\begin{aligned}
& |u|=1 \text {. So } \sup \{\operatorname{Re} w \mid w \in \sigma(u S)\}=r(u S)(=r(S)) \text { and by (9) we get } \\
& r(u S)=\limsup _{0<t \rightarrow \infty} \frac{\log \|\exp (t(u S))\|}{t} \leq \limsup _{z \in \mathbb{C},|z| \rightarrow \infty} \frac{\log \|\exp (z u S)\|}{|z|} \text {. }
\end{aligned}
$$

This implies the first inequality of (8), since $|u|=1$ and $r(u S)=r(S)$.

Now we can prove the main result of this section.

3.9. TheOrem. As above, let $\mathcal{G}$ be a complex finite-dimensional solvable Lie subalgebra of $\mathcal{B}(\mathcal{X}),(\mathcal{T}, \mu)$ be a finite measure space and $\theta$ be a bounded measurable $\mathcal{G}$-valued function on $(\mathcal{T}, \mu)$. For every $p \in[1, \infty]$ we have

$$
r_{p}(\theta) \leq \overline{\mathfrak{r}}_{p}(\theta) \leq \varrho_{p}(\theta) .
$$

Proof. We have $\overline{\mathfrak{r}}_{p}(\theta) \leq \varrho_{p}(\theta)$ by Proposition 2.6 and Remark $2.1\left(2^{\circ}\right)$. To prove $r_{p}(\theta) \leq \overline{\mathfrak{r}}_{p}(\theta)$, let $q \in[1, \infty], 1 / p+1 / q=1$. Let $\varepsilon>0$ be arbitrary and choose $\psi \in L^{q}(\mathcal{T}, \mu)$ as in Lemma 3.6 above. Then by Lemma 3.8 we get

$$
\begin{aligned}
r_{p}(\theta)-\varepsilon & \leq r\left(\int_{\mathcal{T}} \psi \theta d \mu\right)=\limsup _{|z| \rightarrow \infty} \frac{\log \left\|\exp \left(z \int_{\mathcal{T}} \psi \theta d \mu\right)\right\|}{|z|} \\
& =\limsup _{|z| \rightarrow \infty} \frac{\log \left\|\exp \left(\int_{\mathcal{T}} z \psi \theta d \mu\right)\right\|}{\|z \psi\|_{q}}
\end{aligned}
$$

because $\|\psi\|_{q}=1$. Consequently,

$$
r_{p}(\theta)-\varepsilon \leq \limsup _{\varphi \in L^{q}(\mathcal{T}, \mu),\|\varphi\|_{q} \rightarrow \infty} \frac{\log \left\|\exp \left(\int_{\mathcal{T}} \varphi \theta d \mu\right)\right\|}{\|\varphi\|_{q}} \quad\left(=\overline{\mathfrak{r}}_{p}(\theta)\right),
$$

and the desired inequality $r_{p}(\theta) \leq \overline{\mathfrak{r}}_{p}(\theta)$ follows at once since $\varepsilon>0$ was arbitrary.

Now we can obtain the inequalities $(* * *)$ from the Introduction. To this end we specialize Definitions 3.1 and 3.4 in the following way.

3.10. Definition. Let $T=\left(T_{1}, \ldots, T_{n}\right) \in \mathcal{B}(\mathcal{X})^{n}$ be a system of operators generating a finite-dimensional solvable Lie subalgebra $\mathcal{L}$ of $\mathcal{B}(\mathcal{X})$. We define the Cartan-Taylor joint spectrum of $T$ by

$$
\sigma(T):=\left\{\left(\lambda\left(T_{1}\right), \ldots, \lambda\left(T_{n}\right)\right) \mid \lambda \in \Sigma(\mathcal{L})\right\} \quad\left(\subset \mathbb{C}^{n}\right),
$$

where $\Sigma(\mathcal{L})$ is the spectrum of $\mathcal{L}$ (cf. [1]). Moreover, for $p \in[1, \infty]$, we define the geometric spectral radius of $T$ by

$$
r_{p}(T):=\sup \left\{\|z\|_{p} \mid z \in \sigma(T)\right\},
$$

where $\|\cdot\|_{p}$ is the usual $l^{p}$-norm on $\mathbb{C}^{n}$.

Obviously Definition 3.1 reduces to Definition 3.10 when $\mathcal{T}=\{1, \ldots, n\}$, $\mu$ is the counting measure on $\mathcal{T}$ and $\theta(j)=T_{j}$ for $j=1, \ldots, n$. The same 
special case of Theorem 3.9 coincides with the inequalities $(* * *)$ from the Introduction. More precisely we have:

3.11. Corollary. If $T \in \mathcal{B}(\mathcal{X})^{n}$ is a system of operators generating a finite-dimensional solvable Lie subalgebra of $\mathcal{B}(\mathcal{X})$, then for every $p \in[1, \infty]$ we have

$$
r_{p}(T) \leq \overline{\mathfrak{r}}_{p}(T) \leq \varrho_{p}(T) .
$$

4. Situations when the three spectral radii coincide. We are going to prove the following result describing situations when, in the framework of Theorem 3.9, the geometric, analytic and algebraic spectral radii coincide.

4.1. Theorem. Let $(\mathcal{T}, \mu)$ be a finite measure space and $\theta: \mathcal{T} \rightarrow \mathcal{B}(\mathcal{X})$ be a bounded function such that there exists a finite-dimensional solvable Lie subalgebra $\mathcal{G}$ of $\mathcal{B}(\mathcal{X})$ with $\{\theta(t) \mid t \in \mathcal{T}\} \subset \mathcal{G}$. Suppose that one of the following conditions is fulfilled:

$1^{\circ} \mathcal{T}$ is a finite set, $\mu$ is the counting measure on $\mathcal{T}, 1 \leq p \leq \infty$ and either

(a) $\mathcal{G}$ can be chosen nilpotent and $\operatorname{dim} \mathcal{X}<\infty$, or

(b) $\mathcal{G}$ can be chosen abelian (i.e., the values of $\theta$ are mutually commuting),

$2^{\circ} \mathcal{T}$ is a compact separable topological space, $\mu$ is a positive Radon measure on $\mathcal{T}, \theta$ is continuous with respect to the norm operator topology on $\mathcal{B}(\mathcal{X}), \theta(t) \in \mathcal{B}(\mathcal{X})$ is a compact operator for every $t \in \mathcal{T}$ and $p=\infty$,

$3^{\circ} \mathcal{X}$ is a Hilbert space, $\mathcal{G}$ can be chosen nilpotent, $\theta$ is measurable and $p=2$.

Then

$$
r_{p}(\theta)=\overline{\mathfrak{r}}_{p}(\theta)=\varrho_{p}(\theta) .
$$

Proof. In view of Theorem 3.9, it suffices to prove that $r_{p}(\theta)=\varrho_{p}(\theta)$ under each of the hypotheses $1^{\circ}-3^{\circ}$.

If $1^{\circ}$ holds then $r_{p}(\theta)=\varrho_{p}(\theta)$ by either the main result of [7] (in the case (a)), or Theorem 4 of [13] (in the case (b)).

Now assume that $2^{\circ}$ holds. Replacing $\mathcal{G}$ by the Lie algebra generated by $\{\theta(t) \mid t \in \mathcal{T}\}$, we may assume that $\mathcal{G}$ is a finite-dimensional solvable Lie algebra of compact operators. Then $\mathcal{G}$ is triangularizable by Theorem $7^{\prime}$ of [18] (see also Lemma 3.10 in [1]). Since $\theta$ is continuous and $\mathcal{T}$ is a compact space, $\{\theta(t) \mid t \in \mathcal{T}\}$ is a compact subset of $\mathcal{G}$. Hence it is a compact set of simultaneously triangularizable compact operators and by Corollary 4.8 of [21] we get

$$
\varrho_{\infty}(\theta)=\sup _{t \in \mathcal{T}} r(\theta(t))
$$


On the other hand, by Definitions 3.1 and 3.4 we have

$$
r_{\infty}(\theta)=\sup _{\lambda \in \Sigma(\mathcal{G})}\|\lambda \circ \theta\|_{\infty} .
$$

But $\lambda \circ \theta: \mathcal{T} \rightarrow \mathbb{C}$ is a continuous function for every $\lambda \in \Sigma(\mathcal{G})$ (since both $\lambda$ and $\theta$ are continuous), so

$$
r_{\infty}(\theta)=\sup _{\lambda \in \Sigma(\mathcal{G})} \sup _{t \in \mathcal{T}}|(\lambda \circ \theta)(t)| .
$$

Consequently,

$$
r_{\infty}(\theta)=\sup _{t \in \mathcal{T}} \sup _{\lambda \in \Sigma(\mathcal{G})}|\lambda(\theta(t))|=\sup _{t \in \mathcal{T}} \sup _{z \in \sigma(\theta(t))}|z|,
$$

since $\sigma(\theta(t))=\{\lambda(\theta(t)) \mid \lambda \in \Sigma(\mathcal{G})\}$ by Corollary 2.7 of [1]. (Recall that $\theta(t) \in \mathcal{G}$ for $t \in \mathcal{T}$.) Now (10) and (11) together imply $r_{\infty}(\theta)=\varrho_{\infty}(\theta)$.

Finally, assume that $3^{\circ}$ holds. For $A \in \mathcal{B}(\mathcal{X})$ fixed for the moment, let $\mathcal{V}_{A}$ $(\subseteq \mathcal{B}(\mathcal{X}))$ be the complex vector space spanned by the operators $G^{*} A H$ with $G, H \in \mathcal{G}$. Obviously $\operatorname{dim} \mathcal{V}_{A} \leq 2 \operatorname{dim} \mathcal{G}<\infty$ and the $\mathcal{V}_{A}$-valued function $t \mapsto(\theta(t))^{*} A \theta(t)$ is bounded and measurable on the measure space $(\mathcal{T}, \mu)$. Define

$$
\mathcal{M}(A):=\int_{\mathcal{T}}(\theta(t))^{*} A \theta(t) d \mu(t) .
$$

Obviously $\mathcal{M}: \mathcal{B}(\mathcal{X}) \rightarrow \mathcal{B}(\mathcal{X}), A \mapsto \mathcal{M}(A)$, is a bounded linear operator. Moreover it is easy to check that $\mathcal{M}$ is a completely positive map. More generally, for every positive integer $k$ and every $A \in \mathcal{B}(\mathcal{X})$, the values of the function $t \mapsto\left(\theta_{[k]}(t)\right)^{*} A \theta_{[k]}(t)$ are contained in some finite-dimensional vector subspace of $\mathcal{B}(\mathcal{X})$ and this function is bounded and measurable on the measure space $(\mathcal{T} \times \ldots \times \mathcal{T}, \mu \otimes \ldots \otimes \mu)$ (see the notations from the beginning of Section 2). It is easily seen that

$$
\mathcal{M}^{k}(A)=\int_{\mathcal{T} \times \ldots \times \mathcal{T}}\left(\theta_{[k]}(\cdot)\right)^{*} A \theta_{[k]}(\cdot) d(\mu \otimes \ldots \otimes \mu), \quad A \in \mathcal{B}(\mathcal{X}),
$$

and $\mathcal{M}^{k}: \mathcal{B}(\mathcal{X}) \rightarrow \mathcal{B}(\mathcal{X})$ is a completely positive map. Hence

$$
\left\|\mathcal{M}^{k}\right\|=\left\|\mathcal{M}^{k}\left(I_{\mathcal{X}}\right)\right\|=\left\|\int_{\mathcal{T} \times \ldots \times \mathcal{T}}\left(\theta_{[k]}(\cdot)\right)^{*} \theta_{[k]}(\cdot) d(\mu \otimes \ldots \otimes \mu)\right\|,
$$

where $I_{\mathcal{X}}$ is the identity operator on $\mathcal{X}$. Since the last integral defines a positive operator $B_{k}$ (as is easily checked), we have

$$
\begin{aligned}
\left\|B_{k}\right\| & =\sup _{\|x\|=1}\left\langle B_{k} x, x\right\rangle=\sup _{\|x\|=1} \int_{\mathcal{T} \times \ldots \times \mathcal{T}}\left\|\theta_{[k]}(\cdot) x\right\|^{2} d(\mu \otimes \ldots \otimes \mu) \\
& =\sup _{\|x\|=1}\left\|\theta_{[k]}(\cdot) x\right\|_{2}^{2} .
\end{aligned}
$$


Hence we can compute the spectral radius of $\mathcal{M}$ as follows:

$$
r(\mathcal{M})=\lim _{k \rightarrow \infty}\left\|\mathcal{M}^{k}\right\|^{1 / k}=\lim _{k \rightarrow \infty}\left\|B_{k}\right\|^{1 / k}=\left(\varrho_{2}(\theta)\right)^{2},
$$

by (12) and Definition 2.3.

On the other hand, we have

$$
\sigma(\mathcal{M})=\left\{\|\zeta\|_{2}^{2} \mid \zeta \in \sigma(\theta)\right\},
$$

so $r(\mathcal{M})=\left(r_{2}(\theta)\right)^{2}$ by Definition 3.4. Then by (13) we get $r_{2}(\theta)=\varrho_{2}(\theta)$, as desired.

Now, to prove (14) one uses Lemma 3.5. To this end denote by $\mathcal{G}^{\text {op }}$ the opposite Lie algebra of $\mathcal{G}$ (i.e. $\mathcal{G}^{\text {op }}$ coincides with $\mathcal{G}$ as a vector space and has the bracket $[\cdot, \cdot]^{\text {op }}$ defined by $\left.[T, S]^{\text {op }}:=-[T, S]\right)$. Set $\mathcal{Z}:=\mathcal{B}(\mathcal{X})$ and consider the representation

$$
\varrho: \mathcal{G}^{\mathrm{op}} \times \mathcal{G}^{\mathrm{op}} \rightarrow \mathcal{B}(\mathcal{Z}), \quad(T, S) \mapsto L_{T^{*}}+R_{S},
$$

where $L_{T^{*}}(A)=T^{*} A, R_{S}(A)=A S$ for $T, S \in \mathcal{G}^{\text {op }}$ and $A \in \mathcal{B}(\mathcal{X})$. For the spectrum of this representation $([15])$ we have

$$
\sigma(\varrho)=\left\{\bar{\lambda} \otimes \lambda \mid \lambda \in \sigma\left(\operatorname{id}_{\mathcal{G}}\right)\right\}
$$

where $(\bar{\lambda} \otimes \lambda)(T, S)=\overline{\lambda(T)}+\lambda(S)$ for $T, S \in \mathcal{G}^{\text {op }}$ and $\lambda \in \sigma\left(\operatorname{id}_{\mathcal{G}}\right)$ (see [5], [6] and Theorem 3.6.7 of [15]). Here, of course, we denote by $\operatorname{id}_{\mathcal{G}}$ the natural inclusion map of the vector space $\mathcal{G}$ in $\mathcal{B}(\mathcal{X})$, which can be viewed as a representation of $\mathcal{G}$. Let $\mathcal{E}:=\mathcal{G}^{\text {op }} \times \mathcal{G}^{\text {op }}$ and

$$
\omega: \mathcal{T} \rightarrow U(\mathcal{E})=U\left(\mathcal{G}^{\mathrm{op}}\right) \otimes U\left(\mathcal{G}^{\mathrm{op}}\right), \quad t \mapsto \theta(t) \otimes \theta(t) .
$$

Then the values of $\omega$ belong to the finite-dimensional subspace $\mathcal{G}^{\mathrm{op}} \otimes \mathcal{G}^{\mathrm{op}}$ of $U(\mathcal{E})$ and it is easily seen that

$$
\mathcal{M}=\int_{\mathcal{T}} \varrho \circ \omega d \mu .
$$

Hence by Lemma 3.5(b) we have

$$
\sigma(\mathcal{M})=\left\{\int_{\mathcal{T}} \tilde{\lambda} \circ \omega \mid \tilde{\lambda} \in \sigma(\varrho)\right\} \stackrel{(15)}{=}\left\{\int_{\mathcal{T}}(\bar{\lambda} \circ \theta) \cdot(\lambda \circ \theta) d \mu \mid \lambda \in \sigma\left(\operatorname{id}_{\mathcal{G}}\right)\right\},
$$

so

$$
\sigma(\mathcal{M})=\left\{\int_{\mathcal{T}}|\lambda \circ \theta|^{2} \mid \lambda \in \Sigma(\mathcal{G})\right\} .
$$

(Recall that $\Sigma(\mathcal{G})=\sigma\left(\mathrm{id}_{\mathcal{G}}\right)$ since $\mathcal{G}$ is nilpotent; see [1].) Now (14) follows from Definition 3.1.

4.2. Remark. The proof in case $2^{\circ}$ is suggested by the proof of Corollary 1.3 of [10], and the proof in case $3^{\circ}$ extends the proof of Theorem 2 of [4]. 


\section{References}

[1] D. Beltiţă, Spectrum for a solvable Lie algebra of operators, Studia Math. 135 (1999), 163-178.

[2] - Spectral conditions for the nilpotency of Lie algebras, J. Operator Theory, to appear.

[3] D. Beltiţă and M. Şabac, Lie Algebras of Bounded Operators, Birkhäuser, to appear.

[4] R. Bhatia and T. Bhattacharyya, On the joint spectral radius of commuting matrices, Studia Math. 114 (1995), 29-38.

[5] E. Boasso, Dual properties and joint spectra for solvable Lie algebras of operators, J. Operator Theory 33 (1995), 105-116.

[6] -, Tensor products and joint spectra for solvable Lie algebras of operators, Collect. Math. 49 (1998), 9-16.

[7] -, On the joint spectral radius of a nilpotent Lie algebra of matrices, Studia Math. 132 (1999), 15-27.

[8] F. F. Bonsall and J. Duncan, Numerical Ranges of Operators on Normed Spaces and of Elements of Normed Algebras, London Math. Soc. Lecture Note Ser. 2, Cambridge Univ. Press, London, 1971.

[9] M. Chō and W. Żelazko, On geometric spectral radius of commuting $n$-tuples of operators, Hokkaido Math. J. 21 (1992), 251-258.

[10] A. A. Dosiev, Holomorphic functions of a basis in a nilpotent Lie algebra, Funktsional. Anal. i Prilozhen. 34 (2000), no. 4, 82-84 (in Russian).

[11] R. Drnovšek, On reducibility of semigroups of compact operators, Proc. Amer. Math. Soc. 125 (1997), 2391-2394.

[12] A. S. Fainshtein, Taylor joint spectrum for families of operators generating nilpotent Lie algebras, J. Operator Theory 29 (1993), 3-27.

[13] V. Müller, On the joint spectral radius, Ann. Polon. Math. 66 (1997), 173-182.

[14] V. Müller and A. Sołtysiak, Spectral radius formula for commuting Hilbert space operators, Studia Math. 103 (1992), 329-333.

[15] C. Ott, Gemeinsame Spektren für auflösbare Operator-Liealgebren, Dissertation, Kiel, 1997 (http://analysis.math.uni-kiel.de/wrobel/).

[16] P. Rosenthal and A. Sołtysiak, Formulas for the joint spectral radius of non-commuting Banach algebra elements, Proc. Amer. Math. Soc. 123 (1995), 2705-2708.

[17] G.-C. Rota and G. Strang, A note on joint spectral radius, Indag. Math. 22 (1960), 379-381.

[18] M. Şabac, Irreducible representations of infinite dimensional Lie algebras, J. Funct. Anal. 52 (1983), 303-314.

[19] G. Schlüchtermann, A note on multiplication of strong operator measurable functions, Proc. Amer. Math. Soc. 123 (1995), 2815-2816.

[20] V. S. Shul'man and Yu. V. Turovskiı̌, Joint spectral radius and invariant subspaces, Funktsional. Anal. i Prilozhen. 34 (2000), no. 2, 91-94 (in Russian); English transl.: Funct. Anal. Appl. 34 (2000), 156-158.

[21] - - - Joint spectral radius, operator semigroups, and a problem of W. Wojtyński, J. Funct. Anal. 177 (2000), 383-441.

[22] Z. Słodkowski and W. Żelazko, On joint spectra of commuting families of operators, Studia Math. 50 (1974), 127-148.

[23] A. Soltysiak, On the joint spectral radii of commuting Banach algebra elements, ibid. 105 (1993), 93-99.

[24] Yu. V. Turovskiǔ, Volterra semigroups have invariant subspaces, J. Funct. Anal. 162 (1999), 313-322. 
[25] Yu. V. Turovskiı̌, private communication, June 3, 1999.

Institute of Mathematics

"Simion Stoilow"

of the Romanian Academy

P.O. Box 1-764

RO-70700 Bucureşti, Romania

E-mail: dbeltita@imar.ro

Received November 13, 1999

Revised version August 28, 2000 